\title{
Metabolic syndrome is linked to a mild elevation in liver aminotransferases in diabetic patients with undetectable non-alcoholic fatty liver disease by ultrasound
}

Alireza Esteghamati ${ }^{1 *}$, Arsia Jamali ${ }^{1}$, Omid Khalilzadeh ${ }^{1,2}$, Sina Noshad ${ }^{1}$, Mohammad Khalili $^{1}$, Ali Zandieh ${ }^{1}$, Afsaneh Morteza', Manouchehr Nakhjavani ${ }^{1}$

\begin{abstract}
Background: Despite ongoing findings on the relationship between elevated levels of alanine and aspartate aminotransferases (ALT and AST) and metabolic syndrome (MetS), this association in diabetic patients without a known cause for liver enzymes elevation other than diabetes, per se, remains unclear. In this study, we aimed to assess the relationship between circulating liver enzymes and MetS in a relatively large sample of patients with diabetes.

Methods: A total of 670 diabetic patients, without known causes of hepatocellular injury, were enrolled. Patients with ultrasonographic signs of fatty liver disease were not included. Fasting blood samples were obtained and biochemical characteristics were measured. MetS was defined according to the international diabetes federation criteria.

Results: Serum ALT and AST were significantly higher in patients with MetS $(p<0.001)$. High waist circumference and low HDL-cholesterol were significantly associated with elevated ALT (OR $=2.56$ and 2.0, respectively) and AST $(\mathrm{OR}=2.23$ and 2.21, respectively). ALT and AST were significantly associated with MetS $(\mathrm{OR}=2.17$ and 2.31, respectively). These associations remained significant after multiple adjustments for age, sex, BMI, diabetes duration, $\mathrm{HbA1c}$ and medications. There was a significant $(p<0.01)$ positive association between the number of the MetS features and the level of ALT or AST.

Conclusion: In diabetic patients without ultrasonographic evidence of fatty liver, elevated aminotransferases are independently associated with MetS. Despite negative ultrasound results in diabetic patients with MetS, the serum level of liver aminotransferases may be elevated and should be more thoroughly monitored.
\end{abstract}

\section{Introduction}

Insulin insensitivity is a known cause of liver damage [1]. Elevation of circulating liver enzymes including aspartate aminotransferase (AST), alanine aminotransferase (ALT), and alkaline phosphatase (ALP) is suggestive of hepatocellular injury [2-5]. There is increasing evidence that ALT is significantly and independently associated with

\footnotetext{
* Correspondence: esteghamati@tums.ac.ir

${ }^{1}$ Endocrinology and Metabolism Research Center (EMRC), Vali-Asr Hospital,

School of Medicine, Tehran University of Medical Sciences, Tehran, Iran
}

Full list of author information is available at the end of the article type 2 diabetes mellitus [6-8], however not all of the studies support this finding [9].

In recent years, nonalcoholic fatty liver disease (NAFLD), as a novel component of insulin resistance and metabolic syndrome (MetS), has drawn the attention of many researchers. NAFLD encompasses a wide spectrum of liver diseases ranging from simple benign steatosis to steatohepatitis, fibrosis, and cirrhosis [1]. This condition which is associated with long-standing elevations in liver enzymes [10,11], is related to higher risk of adverse cardiovascular events, oxidative stress, endothelial dysfunction, and MetS [12]. 
Despite ongoing findings on the relationship between NAFLD and MetS [13], the relationship between elevated liver enzymes and MetS in diabetic patients without a known cause for liver enzymes elevation other than diabetes, per se, remains unclear. In particular, while most of the studies describe the association between MetS and elevated liver aminotransferases via the NAFLD mechanism [14]; it is not clear what extent of liver steatosis is sufficient to mediate the association between liver enzymes and MetS in diabetes. To best of our knowledge, no evidence is available regarding the association between MetS and serum aminotransferases in patients with mild stages of liver steatosis. To determine subjects with mild liver steatosis from those with advanced stages, ultrasonography is an appropriate screening tool. Ultrasonography with the sensitivity of 60-89 percent and specificity of 66-93 percent in detecting steatosis is proved as a good tool for detection of clinically significant fatty infiltrations, in epidemiologic studies [15]. It is reported that individuals with negative fatty liver changes in ultrasonography have hepatic fat $<30 \%$ [16].

Of note, there is limited evidence regarding the pattern of abnormality in liver enzymes in diabetic patients with and without MetS. In this study, we aimed to assess the relationship between circulating liver enzymes and MetS in a relatively large sample of Iranian patients with type 2 diabetes, after excluding patients with ultrasonographic signs of NAFLD or any other known causes of hepatocellular injury.

\section{Methods}

\section{Study population}

The study population consisted of 670 diabetic subjects who consecutively visited Vali-Asr hospital outpatient diabetes clinic (Tehran, Iran) from June 2007 to September 2009. Diabetes was diagnosed according to American Diabetes Association (ADA) criteria [17]. The study population was divided into two groups of diabetic patients with $(\mathrm{n}=502)$ and without MetS $(\mathrm{n}=168)$. MetS was defined according to the IDF criteria using the cutoffs we recently established for waist circumference (WC) in Iranian adults [18]. Subjects with abdominal obesity (WC $>90 \mathrm{~cm}$ for both men and women) plus at least two of the risk factors from the IDF criteria including high triglyceride levels (TG $\geq 150 \mathrm{mg} / \mathrm{dL}$ ), low HDL-cholesterol (HDL-C $\leq 40 \mathrm{mg} / \mathrm{dL}$ in men and $\leq 50$ $\mathrm{mg} / \mathrm{dL}$ in women), high blood pressure (systolic BP $\geq 130 \mathrm{mmHg}$, diastolic $\mathrm{BP} \geq 85 \mathrm{mmHg}$ or treatment of previously diagnosed hypertension), and high fasting plasma glucose level (FPG $\geq 100 \mathrm{mg} / \mathrm{dL}$ or treatment of previously diagnosed diabetes) were considered to have MetS. Since all of our patients were known cases of diabetes, abdominal obesity plus at least one of the above factors was sufficient to establish a clinical diagnosis of MetS in this study.

A thorough history was taken and physical examination was performed by a trained physician for all patients. None of our patients were on insulin or thiazolidinedione treatment. Patients with a history of alcohol consumption, as well as those with history of a known chronic liver disease including autoimmune hepatitis, hemochromatosis, Wilson's disease, primary biliary cirrhosis, and sclerosing cholangitis were not included in the study. No subject had history of taking medications that are commonly attributed to liver enzyme elevations (such as methotrexate, tetracycline, amiodarone, high doses of estrogen, tamoxifen, diclofenac, amoxicillin, valproic acid or steroids). Abdominal ultrasonography for evaluation of liver (Hitachi EUB 405 apparatus equipped with a convex $3.5 \mathrm{MHz}$ probe) was performed in patients, by a single experienced radiologist (to avoid inter-operator discordance) and those with evidence of fatty liver were excluded. Biochemical evaluation including serum ALT and AST was performed in all subjects. All patients with elevated liver enzymes underwent serologic examination for hepatitis B and C viruses, and those with positive results were not included in the study. Auto antibodies (antinuclear antibody and anti-smooth muscle antibody) and biochemical features (including iron studies, ceruloplasmin, and urinary copper) were also measured in patients with elevated liver enzymes and those with abnormal results were not included.

The study was approved by the local ethics committee of Tehran University of Medical Sciences. All subjects were provided with written informed consent and the study was conducted in accordance with Helsinki declaration.

\section{Data collection}

Anthropometric data including age, sex, height, weight and WC were collected. Height was measured with subject standing without shoes by a standard stadiometer and the nearest one centimeter was recorded. Weight was measured with subjects wearing only light clothing standing on a digital scale; the nearest 0.5 kilogram was recorded. WC was measured at the end of a normal expiration, midway between the inferior margin of the ribs and superior border of the iliac crest in a horizontal plane, and was rounded to the nearest $0.1 \mathrm{~cm}$, according to the previously published literature [19]. Body mass index (BMI; $\mathrm{kg} / \mathrm{m}^{2}$ ) was calculated according to the Quetelet equation (weight in kilograms divided by height in square meter) [20]. BP was measured on the right arm with subject having relaxed for at least 5 minutes, via a standard sphygmomanometer of appropriate 
cuff size and was repeated with 10 minutes interval. The average of these two measurements was recorded for systolic and diastolic BP.

\section{Laboratory methods}

Venous blood samples were collected following an overnight 12-hours of fasting and HbA1c, TG, cholesterol (Chol), HDL-C, low density lipoprotein-cholesterol (LDL-C), fasting plasma glucose (FPG), AST, ALT and ALP were measured. FPG measurement was carried out using the glucose oxidase method. Chol, TG, LDL-C, and HDL-C were determined using enzymatic methods. Analyses of serum ALT, AST and ALP were performed using enzymatic photometry. ALT level $>30 \mathrm{U} / \mathrm{L}$ in women and $>40 \mathrm{U} / \mathrm{L}$ in men, AST level $>30 \mathrm{U} / \mathrm{L}$ in women and $>36 \mathrm{U} / \mathrm{L}$ in men and ALP levels of greater than $306 \mathrm{U} / \mathrm{L}$ in both women and men were considered elevated according to the manufacturer's instructions. Serum ALT and AST were measured by the IFCC (International Federation of Clinical Chemistry and Laboratory Medicine) method (ALT intra-assay coefficient of variation $[\mathrm{CV}]=3.7 \%$, AST intra-assay $\mathrm{CV}=$ $2.5 \%)$. Serum ALP was measured using the DGKC (Deutsche Gesellschaft für Klinische Chemie) method (Intra-assay $\mathrm{CV}=1.5 \%$ ). These measurements were conducted using commercial Parsazmun kits (Tehran, Iran) and a Hitachi 704 automatic analyzer (Tokyo, Japan) [21]. HbA1c was measured using the high performance liquid chromatography method. For diagnosis of hepatitis $B$ and $C$, antibodies to hepatitis $C$ virus, hepatitis $B$ surface antigen, hepatitis $B$ surface antibody, hepatitis $B$ core antibody, hepatitis B e antigen, and hepatitis B e antibody were measured using commercially available enzyme linked immunosorbent assay (ELISA) kits (DRG Diagnostics $\mathrm{GmbH}$, Germany).

\section{Statistical analysis}

All statistical analyses were performed using SPSS software version 17.0 for windows (SPSS Inc. Chicago, IL, USA). Continuous variables are expressed as mean \pm standard deviation. Independent sample t-test was used to identify differences of continuous variables between groups with and without MetS. Categorical variables were compared using Chi square analysis. The degree of association between continuous variables was assessed via Pearson's correlation coefficient (r). Logistic regression analysis was performed to assess the independent correlations between different features of MetS, and ALT or AST. General linear models were used to compare the adjusted mean values of ALT or AST in patients with different numbers of metabolic abnormalities. $\mathrm{P}$ value $<0.05$ was considered statistically significant.

\section{Results}

A total of 670 diabetic patients without ultrasound evidence of fatty liver, with the mean age of $54.12 \pm 10.49$ years (range: 18-80 years) were included in the study. Females constitute $360(53.7 \%)$ of the patients. The mean and median of diabetes duration in the study population was $5.84 \pm 5.69$ and 5.00 years, respectively. The prevalence of MetS in the patients was $74.9 \%$. About $31.5 \%$ of the patients $(n=204)$ had elevated aminotransferases, 110 had only elevated ALT, 13 had only elevated AST and the remaining 81 patients had both elevated ALT and AST. Elevation in ALT or AST was mild in all our patients (less than 2.5 times elevation compared with upper limit of normal range).

As Table 1 summarizes, there were no significant differences in the age, sex, diabetes duration, HbA1c, FPG, LDL-C, Chol, and ALP levels in the patients with and without MetS. However, BMI, WC, diastolic and systolic BP, TG, HDL-C, AST and ALT levels were higher in patients with MetS. Furthermore, there were no significant differences between patients with and without MetS with respect to use of oral anti-diabetic medications, lipid lowering or antihypertensive agents. Also there were no significant association between elevated ALT/AST and use of oral anti-diabetic medications ( $\mathrm{P}=$ 0.589 and $\mathrm{P}=0.224$ for elevated ALT and elevated AST, respectively), lipid lowering agents $(\mathrm{P}=0.859$ and $\mathrm{P}=$ 0.262 , respectively) or anti-hypertensive medications ( $\mathrm{P}=0.718$ and 0.181 , respectively). Both long term control of glycemic situation measured by HbA1c level, and short term glycemic control quantified by FPG was similar in patients with and without elevated ALT $(\mathrm{P}=0.20$ and 0.69 , respectively).

In univariate analysis, serum ALT level was significantly $(\mathrm{p}<0.05)$ correlated with AST $(\mathrm{r}=0.758)$, age $(\mathrm{r}=0.196)$, diabetes duration $(\mathrm{r}=0.149)$, WC $(\mathrm{r}=$ $0.200)$, TG $(r=0.159)$ and was negatively associated with HDL-C ( $\mathrm{r}=-0.165)$. No significant correlation was observed between ALT and ALP level, BP (systolic or diastolic), HbA1c, FPG, and LDL-C. Similar to ALT, AST was significantly $(\mathrm{p}<0.05)$ correlated with age $(\mathrm{r}=$ $0.129)$, diabetes duration $(r=0.123)$, WC $(r=0.188)$, and was negatively associated with HDL-C $(r=-0.137)$. No significant correlation was observed between AST and TG, ALP, BP (systolic or diastolic), HbA1c, FPG or LDL-C.

As shown in Table 2, patients with elevated ALT had significantly higher prevalence of impaired TG levels. However, after adjustment for various confounding factors the significant association disappeared. In addition, the prevalence of Low HDL-C and high WC was significantly higher in patients with elevated ALT and remained significant even after multiple adjustments $(\mathrm{OR}=2.04$, 
Table 1 Clinical and laboratory characteristics of the study subjects with and without metabolic syndrome (MetS)

\begin{tabular}{|c|c|c|c|}
\hline Variables & Without MetS & With MetS & $P$ value \\
\hline N (\%) & $168(26.6)$ & $502(74.9)$ & - \\
\hline Age (years) & $53.39 \pm 10.34$ & $54.36 \pm 10.54$ & 0.300 \\
\hline Females (n, \%) & $101(60.11)$ & $259(51.59)$ & 0.054 \\
\hline Diabetes duration (years) & $5.94 \pm 6.35$ & $5.81 \pm 5.46$ & 0.800 \\
\hline Body mass index $\left(\mathrm{kg} / \mathrm{m}^{2}\right)$ & $25.96 \pm 3.48$ & $30.78 \pm 4.69$ & $<0.001^{*}$ \\
\hline Waist circumference (cm) & $88.59 \pm 8.43$ & $102.94 \pm 8.67$ & $<0.001^{*}$ \\
\hline Diastolic blood pressure $(\mathrm{mmHg})$ & $78.77 \pm 7.28$ & $81.56 \pm 8.19$ & $<0.001^{*}$ \\
\hline Systolic blood pressure $(\mathrm{mmHg})$ & $122.50 \pm 13.92$ & $130.94 \pm 17.34$ & $<0.001^{*}$ \\
\hline Anti hypertensive medications ${ }^{a}(n, \%)$ & $68(40.50)$ & $200(39.84)$ & 0.917 \\
\hline Anti-Diabetic Medications (n, \%) & & & 0.470 \\
\hline No medication & $0(0)$ & $2(0.01)$ & \\
\hline Glibenclamide & $29(17.8)$ & $71(14.1)$ & \\
\hline Metformin & $34(20.2)$ & $123(24.5)$ & \\
\hline Glibenclamide \& Metformin & $63(37.5)$ & $206(41.0)$ & \\
\hline Lipid Lowering Agents (n, \%) & & & 0.189 \\
\hline No medication & $103(61.31)$ & $271(53.98)$ & \\
\hline Statins & $53(31.55)$ & $174(34.66)$ & \\
\hline Fibrates & $4(2.38)$ & $30(5.97)$ & \\
\hline Statins \& Fibrates & $8(4.76)$ & $22(4.38)$ & \\
\hline HbA1c (\%) & $7.68 \pm 1.92$ & $8.03 \pm 1.77$ & 0.130 \\
\hline Fasting plasma glucose (mg/dL) & $162.86 \pm 58.13$ & $166.49 \pm 53.23$ & 0.445 \\
\hline Triglycerides (mg/dL) & $158.21 \pm 90.03$ & $213.01 \pm 118.73$ & $<0.001^{*}$ \\
\hline $\mathrm{HDL}-\mathrm{C}(\mathrm{mg} / \mathrm{dL})$ & $48.16 \pm 11.26$ & $44.23 \pm 12.60$ & $<0.001^{*}$ \\
\hline LDL-C (mg/dL) & $115.59 \pm 37.77$ & $111.73 \pm 36.05$ & 0.236 \\
\hline Total cholesterol (mg/dL) & $195.08 \pm 44.84$ & $197.29 \pm 45.34$ & 0.583 \\
\hline Alanine aminotransferase $(U / L)$ & $27.30 \pm 15.23$ & $31.76 \pm 18.74$ & $0.006^{*}$ \\
\hline Aspartate aminotransferase $(\mathrm{U} / \mathrm{L})$ & $21.53 \pm 9.58$ & $24.17 \pm 13.84$ & $0.007^{*}$ \\
\hline Alkaline phosphatase (U/L) & $148.44 \pm 92.83$ & $151.95 \pm 77.01$ & 0.653 \\
\hline
\end{tabular}

*P value $<0.05$

Variables are presented as mean \pm standard deviation unless stated otherwise.

${ }^{a}$ Medication used for treatment of hypertension include angiotensin converting enzyme inhibitors, angiotensin receptor blockers, calcium channel blockers and beta blockers.

95\% CI: $1.24-3.38$, and OR $=2.48,95 \%$ CI: $1.20-5.11$, respectively). Elevated levels of AST had no significant association with either high BP or high levels of serum TG. Furthermore, although patients with elevated AST had higher prevalence of low HDL-C and high WC, only HDL-C remained significant after adjustment for age, sex, BMI, diabetes duration, HbA1c, and various medications $(\mathrm{OR}=2.13,95 \% \mathrm{CI}: 1.06-4.31)$. Both elevated ALT and AST had a significant $(\mathrm{p}<0.01)$ association with increase in the number of features of MetS (Figure 1).

\section{Discussion}

This study demonstrates that in a reasonably large sample of patients with diabetes, liver enzymes are significantly higher in patients with MetS, in spite of similar long and short-standing glycemic control indices and similar diabetes duration. We also observed that elevated liver enzymes are associated with age, diabetes duration and features of MetS including serum HDL-C level and WC. Oral anti-diabetic, anti-hypertensive, and lipid lowering agents could minimally influence our observed associations, since there was no significant difference between use of medication in patients with and without MetS and also in patients with elevated and normal liver enzymes.

There are very few studies assessing the association between liver enzymes and MetS in patients with diabetes. Hickman et al (2007), on a sample of 189 patients with diabetes, showed that MetS is associated with an unexplained increase in ALT, despite similar glycemic control in patients with and without MetS [22]. In this study, liver enzymes other than ALT including AST and ALP were not assessed. In another study by Forlani and collegues (2008), it was shown that presence of MetS is a significant predictor of raised ALT [23]. These studies did not evaluate the presence of NAFLD in the participants. In the present study, for the first time, we excluded cases with ultrasonographic signs of NAFLD, focusing on 
Table 2 Features of the metabolic syndrome in patients with and without elevated liver aminotransferases

\begin{tabular}{|c|c|c|c|c|}
\hline \multirow[b]{2}{*}{ Variables (n, \%) } & \multicolumn{2}{|c|}{ Diabetic patients } & \multirow[b]{2}{*}{$\begin{array}{c}\text { Crude OR } \\
(95 \% \mathrm{Cl})\end{array}$} & \multirow[b]{2}{*}{$\begin{array}{l}\text { Adjusted OR } \\
(95 \% \mathrm{Cl})\end{array}$} \\
\hline & $\begin{array}{c}\text { without increased } \\
\text { ALT } \\
(n=479)\end{array}$ & $\begin{array}{l}\text { with increased } \\
\text { ALT } \\
(n=191)\end{array}$ & & \\
\hline High blood pressure & $203(42.4)$ & $91(47.6)$ & $1.24(0.88-1.73)$ & $1.11(0.68-1.80)^{1}$ \\
\hline High triglycerides & $286(59.7)$ & $135(70.7)$ & $1.63(1.13-2.33)^{*}$ & $1.50(0.91-2.48)^{1}$ \\
\hline Low HDL-C & $256(53.4)$ & $133(69.6)$ & $2.00(1.40-2.58)^{* * *}$ & $2.04(1.24-3.38) * * 1$ \\
\hline High waist circumference & $368(26.8)$ & $171(89.5)$ & $2.56(1.53-4.26)^{* * *}$ & $2.48(1.20-5.11) * 1$ \\
\hline \multirow[t]{2}{*}{ MetS } & $341(71.2)$ & $161(84.3)$ & $2.17(1.40-3.36)^{* * *}$ & $2.08(1.12-3.87) * 1$ \\
\hline & $\begin{array}{c}\text { without increased } \\
\text { AST } \\
(n=576)\end{array}$ & $\begin{array}{c}\text { with increased } \\
\text { AST } \\
(n=94)\end{array}$ & & \\
\hline High blood pressure & $249(43.2)$ & $45(47.9)$ & $1.21(0.78-1.87)$ & $1.05(0.56-1.98)^{2}$ \\
\hline High triglycerides & $354(61.4)$ & $67(71.3)$ & $1.56(0.97-2.51)$ & $1.39(0.71-2.73)^{2}$ \\
\hline Low HDL-C & $320(55.6)$ & $69(73.4)$ & $2.21(1.36-3.59)^{* *}$ & $2.13(1.06-4.31) * 2$ \\
\hline High waist circumference & $405(70.3)$ & $84(89.4)$ & $2.23(1.12-4.43)^{*}$ & $2.17(0.89-5.31)^{2}$ \\
\hline MetS & $421(73.1)$ & $81(86.2)$ & $2.31(1.25-4.27)^{* *}$ & $2.23(1.09-4.57) * 2$ \\
\hline
\end{tabular}

${ }^{*} \mathrm{P}<0.05$, **P $<0.01$, ***P $<0.001$.

${ }^{1}$ Adjusted for age, sex, BMl, diabetes duration, $\mathrm{HbA} 1 \mathrm{c}$ and medications (anti diabetic, lipid lowering or antihypertensive agents).

${ }^{2}$ Adjusted for age, sex, BMI, diabetes duration, $\mathrm{HbA} 1 \mathrm{c}$ and medications (anti diabetic, lipid lowering or antihypertensive agents).

the patients with elevated liver enzymes without any known cause, except diabetes, per se. Our results showed an independent association between MetS and mildly elevated AST/ALT in diabetes patients without ultrasound evidence of fatty liver disease. This can be due to a mild underlying steatohepatitis, which is undetectable by ultrasound. Our results can be interpreted in parallel with the findings of the study by Mofrad et al (2003), which showed that even low normal ALT values can be associated with underlying steatohepatitis, especially in diabetic patients [24].

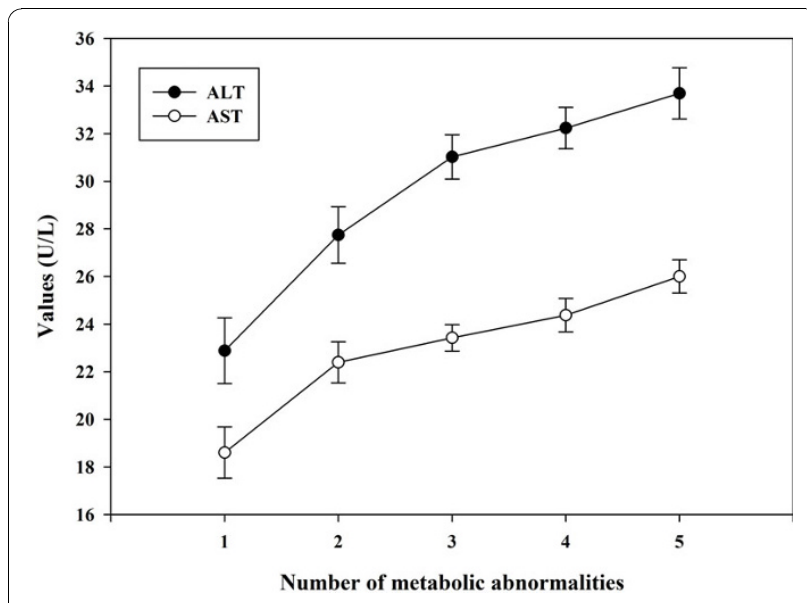

Figure 1 There was a significant $(p<0.01)$ association between the number of the metabolic abnormalities and ALT or AST, after adjustment for age, sex and BMI, HbA1c, diabetes duration and medications.
The pathogenesis of liver damage in patients with diabetes is not thoroughly understood and most of our current knowledge is related to the connection between NAFLD and insulin resistance $[14,25]$. Hepatic insulin resistance can play an important role in liver dysfunction and elevation of liver enzymes $[8,26]$. Also, inflammatory cytokines including tumor necrosis factor and interleukin 6 are proposed in pathogenesis of hepatocellular injury in insulin resistance/MetS [14]. Increased levels of TG and free fatty acids causes accumulation of TG in nonadipose tissues including liver [27] which results in lipid peroxidation. Subsequently, mitochondrial dysfunction caused by formation of toxic metabolites and reactive oxygen species induces cell apoptosis $[14,25,28]$. The association between MetS and liver enzymes in our study shows that in diabetes, MetS is possibly linked to undetectable mild stage of NAFLD. This notifies clinicians to more thoroughly monitor serum aminotransferase in diabetic patients, particularly when the diabetic patient has MetS as well. NAFLD is a feature of MetS [29], and in diabetic patients with MetS, regardless of negative ultrasound results, NAFLD might be present and contribute to the complex of different features of MetS.

This study had potential limitations: first, the crosssectional nature of this study precludes cause and effect relationships. Therefore, further longitudinal studies are paramount to investigate the role of liver enzymes as risk factors of the MetS. Second, it should be noted that liver biopsy is considered as the gold standard of diagnosis of NAFLD [30,31], however it is invasive, expensive, and may bear potential risks [32]. There are some imaging techniques for assessing hepatic fat content. 
Non-enhanced Computed Tomography (CT) can qualitatively detect macrovesicular steatosis of $30 \%$ and more, however there are conflicting evidence on its value in quantifying liver fat content [32]. Enhanced CT is less valuable in assessing liver fat since contrast type, injection rate, and timing can significantly influence the liver to spleen attenuation difference for diagnosing fatty liver $[33,34]$. Several studies have demonstrated an appropriate correlation between severity of steatosis on magnetic resonance imaging (MRI) and histologic biopsies [35-37]. Emerging imaging techniques such as dual gradient echo MRI (DGE-MRI) and proton magnetic resonance spectroscopy $\left({ }^{1} \mathrm{H}\right.$-MRS), are currently evaluated for detecting hepatic steatosis. One study has demonstrated DEG-MRI is highly accurate for evaluating moderate to severe hepatic steatosis (sensitivity and specificity of greater than $90 \%$ ) and fairly sensitive (sensitivity $=76.7 \%)$ and specific $($ specificity $=87.1 \%)$ for detection of all degrees of steatosis [38]. According to our current knowledge, most studies have shown that ${ }^{1} \mathrm{H}$-MRS is the method of choice for detecting steatosis, with a high correlation between the fat fraction estimated by this method $(r=0.70$ and 0.71$)$ and histologic evaluation [38-40]. Nevertheless, we believe that for the purpose of this epidemiologic study, ultrasonography is an appropriate screening tool for determining negative or mild stages of fatty liver disease, with a reasonable accuracy [15]. Our study provided significant insight to our knowledge on the association between elevated liver enzymes and MetS in diabetic patients with mild stages of fatty liver, undetectable in ultrasound. An interesting topic, which needs further attention in future studies, is the significance of serum gamma-glutamyl transpeptidase (GTP) in diabetic patients, and its association with MetS. It is interesting to compare GTP with AST or ALT in prediction of MetS in a diabetes status.

In conclusion, we showed an independent association between elevated ALT/AST and MetS in diabetic patients with undetectable mild stages of NAFLD. Despite negative ultrasound results, the serum level of liver aminotransferases may be elevated in diabetic patients with MetS and should be more thoroughly monitored.

\section{Conflict of interests}

The authors declare that they have no competing interests.

\footnotetext{
Author details

${ }^{1}$ Endocrinology and Metabolism Research Center (EMRC), Vali-Asr Hospital, School of Medicine, Tehran University of Medical Sciences, Tehran, Iran. ${ }^{2}$ Advanced Diagnostic and Interventional Radiology Research Center (ADIR), Imaging Medical Center, Imam Hospital, Tehran University of Medical Sciences, Tehran, Iran.
}

\section{Authors' contributions}

AE conceived the study, participated in its design, coordination and acquisition of data. AJ wrote the first draft and contributed to the statistical analysis. OK participated in manuscript writing, statistical analysis and acquisition of data. SN and MK helped in drafting the manuscript, statistical analysis and interpretation of the results. AZ participated in acquisition of data, writing and editing the manuscript. AM helped to perform the statistical analysis and participated in writing. MN participated in interpretation of the results and editing the manuscript. All authors read and approved the final manuscript.

Received: 13 June 2010 Accepted: 3 November 2010 Published: 3 November 2010

\section{References}

1. Lopez-Bermejo A, Botas P, Funahashi T, Delgado E, Kihara S, Ricart W, Fernandez-Real JM: Adiponectin, hepatocellular dysfunction and insulin sensitivity. Clin Endocrinol (Oxf) 2004, 60(2):256-263.

2. Kew MC: Serum aminotransferase concentration as evidence of hepatocellular damage. Lancet 2000, 355(9204):591-592.

3. Keil E: Determination of enzyme activities in serum for the detection of xenobiotic effects on the liver. Exp Pathol 1990, 39(3-4):157-164.

4. Herrera JL: Abnormal liver enzyme levels. The spectrum of causes. Postgrad Med 1993, 93(2):113-116.

5. Sherman KE: Alanine aminotransferase in clinical practice. A review. Arch Intern Med 1991, 151(2):260-265.

6. Vozarova B, Stefan N, Lindsay RS, Saremi A, Pratley RE, Bogardus C, Tataranni PA: High alanine aminotransferase is associated with decreased hepatic insulin sensitivity and predicts the development of type 2 diabetes. Diabetes 2002, 51(6):1889-1895.

7. Sattar N, Scherbakova O, Ford I, O'Reilly DS, Stanley A, Forrest E, Macfarlane PW, Packard CJ, Cobbe SM, Shepherd J: Elevated alanine aminotransferase predicts new-onset type 2 diabetes independently of classical risk factors, metabolic syndrome, and C-reactive protein in the west of Scotland coronary prevention study. Diabetes 2004, 53(11):2855-2860.

8. Hanley AJ, Williams K, Festa A, Wagenknecht LE, D'Agostino RB, Kempf J, Zinman B, Haffner SM: Elevations in markers of liver injury and risk of type 2 diabetes: the insulin resistance atherosclerosis study. Diabetes 2004, 53(10):2623-2632.

9. Nakanishi N, Suzuki K, Tatara K: Serum gamma-glutamyltransferase and risk of metabolic syndrome and type 2 diabetes in middle-aged Japanese men. Diabetes Care 2004, 27(6):1427-1432.

10. Clark JM, Diehl AM: Nonalcoholic fatty liver disease: an underrecognized cause of cryptogenic cirrhosis. JAMA 2003, 289(22):3000-3004.

11. Clark JM, Brancati FL, Diehl AM: The prevalence and etiology of elevated aminotransferase levels in the United States. Am J Gastroenterol 2003, 98(5):960-967.

12. Musso G, Gambino R, Bo S, Uberti B, Biroli G, Pagano G, Cassader M: Should nonalcoholic fatty liver disease be included in the definition of metabolic syndrome? A cross-sectional comparison with Adult Treatment Panel III criteria in nonobese nondiabetic subjects. Diabetes Care 2008, 31(3):562-568

13. Stein $L L$, Dong $M H$, Loomba $R$ : Insulin sensitizers in nonalcoholic fatty liver disease and steatohepatitis: Current status. Adv Ther 2009, 26(10):893-907.

14. Edmison J, McCullough AJ: Pathogenesis of non-alcoholic steatohepatitis: human data. Clin Liver Dis 2007, 11(1):75-104, ix.

15. Angulo P: Nonalcoholic fatty liver disease. N Engl J Med 2002, 346(16):1221-1231.

16. Torres DM, Harrison SA: Diagnosis and therapy of nonalcoholic steatohepatitis. Gastroenterology 2008, 134(6):1682-1698.

17. Diagnosis and classification of diabetes mellitus. Diabetes Care 2008, 31(Suppl 1(1)):S55-60.

18. Esteghamati A, Abbasi M, Rashidi A, Meysamie A, Khalilzadeh $O$, Haghazali M, Asgari F, Nakhjavani M: Optimal waist circumference cut-offs for the diagnosis of metabolic syndrome in Iranian adults: results of the third national survey of risk factors of non-communicable diseases (SuRFNCD-2007). Diabet Med 2009, 26(7):745-746. 
19. Alberti KG, Zimmet P, Shaw J: Metabolic syndrome-a new world-wide definition. A Consensus Statement from the International Diabetes Federation. Diabet Med 2006, 23(5):469-480.

20. Levey AS, Bosch JP, Lewis JB, Greene T, Rogers N, Roth D: A more accurate method to estimate glomerular filtration rate from serum creatinine: a new prediction equation. Modification of Diet in Renal Disease Study Group. Ann Intern Med 1999, 130(6):461-470.

21. Lentjes EG, Harff GA, Backer ET: Evaluation of the Hitachi 704 automatic analyzer. Clin Chem 1987, 33(11):2089-2092.

22. Hickman IJ, Whitehead JP, Prins JB, Macdonald GA: Raised alanine transaminase and decreased adiponectin are features of the metabolic syndrome in patients with type 2 diabetes. Diabetes Obes Metab 2007, 9(3):438-440.

23. Forlani G, Di Bonito P, Mannucci E, Capaldo B, Genovese S, Orrasch M Scaldaferri L, Di Bartolo P, Melandri P, Dei Cas A, et al: Prevalence of elevated liver enzymes in Type 2 diabetes mellitus and its association with the metabolic syndrome. J Endocrinol Invest 2008, 31(2):146-152.

24. Mofrad P, Contos MJ, Haque M, Sargeant C, Fisher RA, Luketic VA Sterling RK, Shiffman ML, Stravitz RT, Sanyal AJ: Clinical and histologic spectrum of nonalcoholic fatty liver disease associated with normal ALT values. Hepatology 2003, 37(6):1286-1292.

25. Day CP: Pathogenesis of steatohepatitis. Best Pract Res Clin Gastroenterol 2002, 16(5):663-678.

26. Tohidi M, Harati H, Hadaegh F, Mehrabi Y, Azizi F: Association of liver enzymes with incident type 2 diabetes: A nested case control study in an Iranian population. BMC Endocr Disord 2008, 8(5):5.

27. Unger RH: Lipotoxic diseases. Annu Rev Med 2002, 53:319-336.

28. Day CP, Saksena S: Non-alcoholic steatohepatitis: definitions and pathogenesis. J Gastroenterol Hepatol 2002, 17(Suppl 3(3)):S377-384.

29. Marchesini G, Brizi M, Bianchi G, Tomassetti S, Bugianesi E, Lenzi M, McCullough AJ, Natale S, Forlani G, Melchionda N: Nonalcoholic fatty liver disease: a feature of the metabolic syndrome. Diabetes 2001, 50(8):1844-1850

30. Adams LA, Angulo P, KD L: Nonalcoholic fatty liver disease. CMAJ 2005, 172:899-905.

31. Crowley H, Lewis WD, Gordon F, Jenkins R, Khettry U: Steatosis in donor and transplant liver biopsies. Hum Pathol 2000, 31(10):1209-1213.

32. Mehta SR, Thomas EL, Bell JD, Johnston DG, Taylor-Robinson SD: Noninvasive means of measuring hepatic fat content. World I Gastroenterol 2008, 14(22):3476-3483.

33. Jacobs JE, Birnbaum BA, Shapiro MA, Langlotz CP, Slosman F, Rubesin SE, Horii SC: Diagnostic criteria for fatty infiltration of the liver on contrastenhanced helical CT. AJR Am J Roentgenol 1998, 171(3):659-664.

34. Johnston RJ, Stamm ER, Lewin JM, Hendrick RE, Archer PG: Diagnosis of fatty infiltration of the liver on contrast enhanced CT: limitations of liver-minus-spleen attenuation difference measurements. Abdom Imaging 1998, 23(4):409-415.

35. Ma J, Son JB, Zhou Y, Le-Petross H, Choi H: Fast spin-echo triple-echo dixon (fTED) technique for efficient T2-weighted water and fat imaging. Magn Reson Med 2007, 58(1):103-109.

36. Qayyum A, Goh JS, Kakar S, Yeh BM, Merriman RB, Coakley FV: Accuracy of liver fat quantification at MR imaging: comparison of out-of-phase gradient-echo and fat-saturated fast spin-echo techniques-initial experience. Radiology 2005, 237(2):507-511.

37. Pilleul F, Chave G, Dumortier J, Scoazec JY, Valette PJ: Fatty infiltration of the liver. Detection and grading using dual T1 gradient echo sequences on clinical MR system. Gastroenterol Clin Biol 2005, 29(11):1143-1147.

38. Lee SS, Park SH, Kim HJ, Kim SY, Kim MY, Kim DY, Suh DJ, Kim KM, Bae MH, Lee JY, et al: Non-invasive assessment of hepatic steatosis: prospective comparison of the accuracy of imaging examinations. J Hepatol 52(4):579-585.

39. Longo R, Pollesello P, Ricci C, Masutti F, Kvam BJ, Bercich L, Croce LS, Grigolato P, Paoletti S, de Bernard B, et al: Proton MR spectroscopy in quantitative in vivo determination of fat content in human liver steatosis. J Magn Reson Imaging 1995, 5(3):281-285.

40. Thomsen C, Becker U, Winkler K, Christoffersen P, Jensen M, Henriksen O: Quantification of liver fat using magnetic resonance spectroscopy. Magn Reson Imaging 1994, 12(3):487-495. doi:10.1186/1758-5996-2-65

Cite this article as: Esteghamati et al:: Metabolic syndrome is linked to a mild elevation in liver aminotransferases in diabetic patients with undetectable non-alcoholic fatty liver disease by ultrasound. Diabetology \& Metabolic Syndrome 2010 2:65.

\section{Submit your next manuscript to BioMed Central and take full advantage of:}

- Convenient online submission

- Thorough peer review

- No space constraints or color figure charges

- Immediate publication on acceptance

- Inclusion in PubMed, CAS, Scopus and Google Scholar

- Research which is freely available for redistribution

Submit your manuscript at www.biomedcentral.com/submit
Ciomed Central 